\title{
Recurrent Laryngeal Nerve Paralysis
}

National Cancer Institute

\section{Source}

National Cancer Institute. Recurrent Laryngeal Nerve Paralysis. NCI Thesaurus. Code C78592.

Paralysis of the recurrent laryngeal nerve. Causes include surgical and non-surgical traumas, neoplasms (e.g., lung carcinoma), and inflammatory neuritis. 